\title{
eHealth et quotidien clinique: un regard du Nouveau Monde
}

\section{Yvonne Gilli}

Dr méd., membre du Comité central de la FMH, responsable du département Numérisation / eHealth

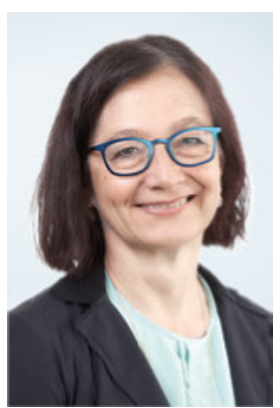

Quelques jours seulement après l'élection du $45^{\mathrm{e}}$ président des Etats-Unis s'est tenu à Chicago le $40^{\mathrm{e}}$ symposium de l'AMIA, l'«American Medical Informatics Association $»^{1}$. Ce symposium offre une plateforme scientifique indépendante pour l'échange d'expériences international et interprofessionnel sur l'informatique médicale, la technologie informatique et les soins de santé.

Avec le dossier électronique du patient, la Suisse veut donner une impulsion au numérique dans le milieu de santé, et le monde politique espère même en retirer un gain d'efficience et une réduction des coûts. Or cet objectif ne pourra pas être atteint sur la base des conditions-cadres actuelles: les incitatifs financiers sont trop faibles pour réunir durablement les systèmes informatiques des cliniques et les solutions individuelles des cabinets. Le retard en matière de connaissances et de formation est bien trop important dans le domaine de l'informatique médicale, alors que celui-ci est aujourd'hui déjà en plein essor aux EtatsUnis et manque même de main d'œuvre.

\section{Nous devons profiter de l'avance d'autres pays pour mettre au point le dossier électronique du patient.}

A l'initiative de la FMH, une délégation du groupe de travail interprofessionnel sur le dossier électronique du patient (IPAG EPD) a pris part au congrès de l'AMIA. Dominik Aronsky² nous a fait visiter deux cliniques de première importance, le Vanderbilt University Medical Center à Nashville et le Partners HealthCare à Boston. Des exposés axés sur la pratique et la visite d'un grand centre d'urgences nous ont sensibilisés au potentiel encore inexploité en Suisse. Le principal message que je retiendrai est simple: nous ne pourrons profiter de l'expérience et de l'avance des Etats-Unis et de certains pays européens qu'en intégrant ces nouvelles connaissances dans nos processus. Car en ce qui concerne la saisie électronique, la gestion et l'échange de données de patients, les défis auxquels nous sommes confrontés sont les mêmes.
Les solutions logicielles et matérielles doivent être conviviales et générer une plus-value. «L'informaticien connaît la valeur des standards pour l'utilisation des données, l'interopérabilité, la stabilité du système et les outils d'aide à la décision. Le clinicien connaît ses propres procédures de travail et ses besoins. C'est en collaborant que chacun atteindra ses objectifs.. ${ }^{3}$ Telle est la conclusion d'un projet de recherche du Vanderbilt Hospital, à l'occasion duquel plus de cinquante infirmiers-chefs en soins aigus, pédiatrie incluse, se sont

\section{Pour trouver des solutions qui fonctionnent, l'informatique doit suivre les procédures cliniques complexes et non l'inverse.}

attelés avec succès à réorganiser le système de documentation électronique pour réduire la charge administrative. Les principaux problèmes identifiés concernent la documentation à double ou inutile et les processus de communication au sein de l'équipe soignante. Avec l'aide de groupes de travail interdépartementaux, ils ont ainsi pu diminuer le temps de documentation d'une demi-heure par soignant et par jour et réduire d'un tiers le volume de documentation par patient. Je peux facilement imaginer que le potentiel inexploité est identique chez les médecins et qu'il serait bénéfique pour plus de satisfaction et davantage de temps en contact direct avec les patients.

Ce n'est là qu'un exemple des défis du quotidien clinique. Les exposés du symposium de l'AMIA comportent plus de deux mille pages et touchent pratiquement tous les thèmes également brûlants pour nous, de la cybermédication au Big Data. Pour trouver des solutions qui fonctionnent, l'informatique doit «suivre» les procédures cliniques complexes et non l'inverse. Les spécialistes en informatique médicale, notamment, sont là pour faire le lien entre ces deux disciplines. Promouvoir la formation, la recherche et la mise en pratique est possible avec des conditions-cadres politiques appropriées, qui coûtent toutefois un peu plus que ce que la Suisse est pour l'instant prête à investir dans un avenir qui changera fondamentalement notre système de santé. 